\title{
Three Versus Seven-Day Treatment of Asymptomatic Bacteriuria in Pregnant Women: A Pilot Investigation
}

\author{
Pooja Mittal, Vivien Pan and Deborah A. Wing*
}

Division of Maternal-Fetal Medicine, Department of Obstetrics and Gynaecology, University of California, Irvine
Medical Center, 101 The City Drive South, Building 56, Suite 800, Orange, CA 92868, USA

Abstract: Objective: To compare 3-day vs 7-day antimicrobial treatment for asymptomatic bacteriuria in pregnant women in a pilot study.

Methods: Pregnant women with asymptomatic bacteriuria $(100,000 \mathrm{cfu} / \mathrm{mL})$ were randomized to 3-day or 7-day nitrofurantoin treatment. Exclusion criteria included symptomatic bacteriuria at enrollment, previously treated bacteriuria in the current pregnancy, underlying renal disorders, antibiotic use within one week prior to initial culture or treatment, bacterial resistance to nitrofurantoin, or other contraindications to nitrofurantoin. Urine test of cure (TOC) was performed 2-4 weeks after initiation of treatment. Subjects with persistent bacteriuria received an additional 7-day treatment course with an antimicrobial agent based on uropathogen sensitivities and selected at the physician's discretion. Following delivery, antenatal, perinatal, and neonatal data were reviewed. Statistical analyses were performed using t-test, Chisquare, and Fisher exact where appropriate. Analysis was based on intent-to-treat.

Results: Forty-four of 60 patients had complete prenatal and delivery records. Twenty-four patients (54.5\%) were randomized to 3 -day treatment and 20 patients $(45.5 \%)$ to 7 -day treatment. Six $(25 \%)$ patients in the 3-day group had persistent bacteriuria at TOC, compared to $2(10 \%)$ patients in the 7 -day group $(\mathrm{p}=0.19)$. There were no statistical differences between the groups in maternal age, gestational age at enrollment, mode of delivery, birth weight, NICU admissions, or the incidence of recurrent urinary tract infections or pyelonephritis.

Conclusion: Based on our small sample group, 3-day nitrofurantoin treatment had statistically comparable outcomes to 7 day treatment for asymptomatic bacteriuria in pregnant patients, yet 3-day-treated women were 2.5 times more likely to have persistent bacteriuria than 7-day-treated women. The possibility of a beta error exists. In light of recent data which indicates that 1-day nitrofurantoin therapy is less effective than 7-day treatment in pregnant women, we recommend 7-day treatment for ASB.

\section{INTRODUCTION}

Asymptomatic bacteriuria (ASB) affects $2-14 \%$ of pregnancies annually in the United States $[1,2]$. This translates to $80,000-400,000$ cases a year [3]. The traditional diagnostic criteria of significant bacteriuria included cultures of 10 colony forming units ( $\mathrm{cfu}) / \mathrm{mL}$ of a single uropathogen on two consecutive clean catch urine specimens $[4,5]$. However, recent evidence suggests that lower colony counts $(>10)$ demonstrate infection that will predispose to the development of pyelonephritis in pregnancy $[1,6,7]$. In pregnancy, the presence of ASB has been found to increase the risk of preterm delivery, infants of low birth weight [8] and other adverse perinatal outcomes [9]. Antibiotic treatment of ASB in pregnancy has been shown to significantly lower the risk of preterm labor when compared to untreated counterparts [10]. Furthermore, pregnant women are at an increased risk for the development of symptomatic urinary tract infections resulting from ASB than their non-pregnant counterparts. It is estimated that $30-50 \%$ of women

*Address correspondence to this author at the Division of Maternal-Fetal Medicine, Department of Obstetrics and Gynaecology, University of California, Irvine Medical Center, 101 The City Drive South, Building 56, Suite 800, Orange, CA 92868, USA; Tel: (714)456-5967;

Fax: (714)456-7091; E-mail: mfm@uci.edu with a diagnosis of ASB in pregnancy will progress to a symptomatic infection [11]. Routine screening and treatment for ASB in pregnancy to prevent progression to pyelonephritis has been shown to be both beneficial and cost-effective [12]. Appropriate treatment of ASB in pregnancy decreases the progression rate of pyelonephritis down to $3 \%$ [13], emphasizing the importance of insuring adequate treatment. Pyelonephritis in pregnancy is associated with an increase in both maternal and fetal morbidities [13-15].

The current prevailing practice for treatment of ASB in pregnancy is a 7-day course of an oral antimicrobial agent [4, $5,16-18]$. There is no consensus on the appropriate first-line therapy for ASB in pregnancy [19]. If bacteriuria persists, a repeat 7 or 14 day course is prescribed. In non-pregnant women, short course antibiotics have become routine in the treatment of even symptomatic bacteriuria, or cystitis [2022]. After a meta-analysis of the literature, the Infectious Disease Society of America (IDSA) issued committee guidelines supporting the efficacy of a 3-day course of an appropriate antimicrobial for treatment of cystitis in nonpregnant women [23]. Short course therapy carries with it the benefits of decreased healthcare costs, fewer side effects, and increased patient compliance [24]. We undertook this 
pilot study to determine the feasibility of the 3-day short course antimicrobial treatment to the pregnant population diagnosed with ASB.

\section{MATERIALS AND METHODOLOGY}

The single institution study was conducted at the Women's and Children's Hospital of the Los Angeles County + University of Southern California Hospital system as well as at Good Samaritan Hospital, both located in Los Angeles, CA. Our intent was to conduct a pilot trial given that at the time of study initiation, there are no previous reports in the literature for short-course treatment of ASB in pregnancy. Approval was obtained from the Institutional Review Board of both institutions and written informed consent obtained from all participants. Subjects were recruited from the prenatal clinics at both hospitals for this prospective randomized controlled trial from 2000 to 2004. Routine urine dipstick, performed at every prenatal visit, was sent for urine culture if suspicious for bacteriuria (positive nitrate, leukocyte esterase, and/or protein). Prior to obtaining urine culture sample, patients underwent education for use of disinfectant wipes and midstream clean-catch collection. Patients, at any gestational age, with a urine culture exhibiting bacterial growth $>100,000 \mathrm{cfu} / \mathrm{ml}$ of a uropathogen and no symptoms of cystitis (dysuria, urgency, frequency, pyrexia) were enrolled. Additional inclusion criteria included pregnant women with a documented intrauterine pregnancy greater than 18 years of age, and the ability to provide informed consent. Exclusion criteria included the following: symptomatic bacteriuria at the time of enrollment, previously treated bacteriuria in the current pregnancy, congenital or acquired urinary tract anomaly, antibiotic use in the week prior to enrollment, glucose-6phosphate dehydrogenase deficiency, allergy to nitrofurantoin, and pathogen insensitive to nitrofurantoin.

At the time of enrollment, initial data collection included maternal demographics (age, gravidity, parity, ethnicity, medical problems), and bacterial species isolated. Subjects were then randomized via a computer generated randomization algorithm to either a 3-day or 7-day antimicrobial course. Assignments were placed in sealed, opaque envelopes until which time a subject was enrolled, and the next envelope opened to make the assignment. The dosing regimens were not blinded due to financial resource limitations. The first line antimicrobial agent used was nitrofurantoin (Macrodantin $₫ 100 \mathrm{mg}$ QID or Macrobid $\AA$ $100 \mathrm{mg}$ BID), chosen for the minimal uropathogen resistance to the drug in our institutions, as well as its high urinary concentration. The choice between one and the other formulation of nitrofurantoin was made at the discretion of the managing physician.

At the subsequent prenatal visit, two to four weeks after initiating treatment, a second midstream urine specimen was collected for repeat urine culture. This served as a test of cure (TOC). Patients with urine cultures that demonstrated persistent bacteriuria (urine culture demonstrating any growth of the same uropathogen) were considered failed treatment and received an additional 7-day course of antimicrobial therapy based on bacterial sensitivities. Secondary outcome measures included comparison of the two treatment groups for persistent or recurrent asymptomatic bacteriuria (after treatment, incidence of symptomatic bacteriuria (cystitis or pyelonephritis), gestational age at delivery, infant birth weight, neonatal intensive care admissions, and incidence of preterm delivery. We diagnosed acute pyelonephritis based on the presence of fever (temperature of 100.4 degrees Fahrenheit), costovertebral angle tenderness, and urinalysis suggestive of infection (positive leukocyte esterase and nitrites). Uropathogens from these cultures were tabulated.

Statistical analyses were performed with the SPSS 13.0 statistical software pack (SPSS Inc., Chicago, IL). Student $t$ test, Chi-square, and Fisher exact test were used where appropriate. A probability value of $<.05$ was considered significant.

\section{RESULTS}

A total of 60 gravid women were enrolled (Fig. 1). Thirty-five subjects were randomized to 3-day treatment, while 25 were randomized to 7-day treatment. Forty-four women completed the trial so that full datasets were available on 24 women in the 3-day arm, and 20 women in the 7-day arm. As can be seen in Table 1, the two groups did not significantly differ in respect to demographic characteristics, [gravidity, parity, gestational age at enrollment, race/ethnicity (data not shown)] with the exception of maternal age where a significant difference was noted between the 3-day and 7-day groups. The distribution of specific uropathogens and their specific colony counts did not differ between the two groups. E. coli was the most prevalent causative organism, cultured in $63.6 \%$ of all initial cultures, followed by E. faecalis (13.6\%), S. agalactiae $(7 \%)$, S. aureus $(7 \%)$.

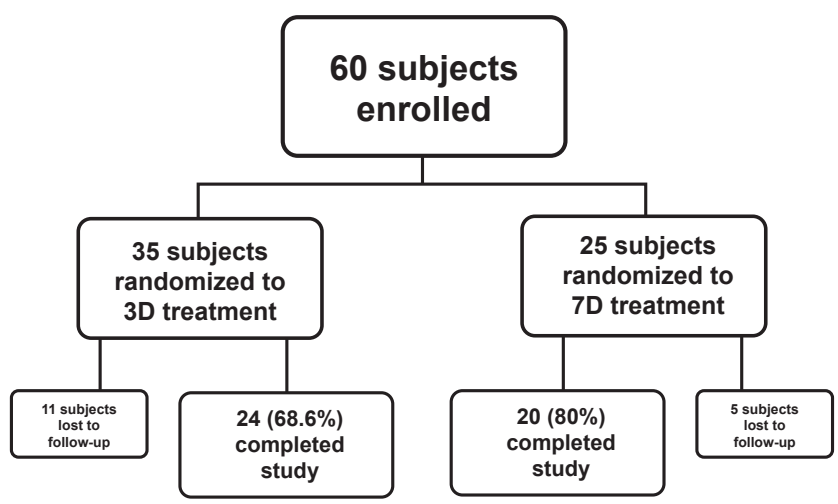

Fig. (1). Participant flow chart for comparison of 3-day vs 7-day nitrofurantoin treatment of asymptomatic bacteriuria in pregnancy.

For the primary outcome measure, persistent bacteriuria on TOC, no statistically significant difference was found between 3-day and 7-day treatment groups; however, those women receiving the 3 -day regimen were 2.5 times more likely to demonstrate persistent bacteriuria than those treated with the 7-day regimen. No difference was noted between the two groups in the number of recurrent infections $(p=0.57)$ or the development of pyelonephritis. For those subjects who developed pyelonephritis, the length of hospital stay (in days) was similar between treatment groups (Table 2). The vast majority of these pyelonephritis cases were due to Escherichia coli $(10 / 11,90.9 \%)$ which was pan-sensitive to standardly administered antibiotics. Of the women who 
developed pyelonephritis, three were found to have persistent bacteriuria after undergoing their initial treatment. These were two from the 3-day group and one from the 7day group.

Table 1. Maternal Characteristics

\begin{tabular}{|l|c|c|c|}
\hline & $\begin{array}{c}\text { 3-Day } \\
\text { (n=35) }\end{array}$ & $\begin{array}{c}\text { 7-Day } \\
\text { (n=25) }\end{array}$ & P-Value \\
\hline \hline Maternal age (years) & $27.86 \pm 6.89$ & $31.76 \pm 6.56$ & 0.03 \\
\hline Gravidity & $2.97 \pm 2.04$ & $3.16 \pm 1.7$ & 0.76 \\
\hline Parity & $1.23 \pm 1.35$ & $1.48 \pm 1.36$ & 0.45 \\
\hline $\begin{array}{l}\text { Gestational age at enrollment } \\
\text { (weeks) }\end{array}$ & $16.8 \pm 8.0$ & $17.6 \pm 8.6$ & 0.77 \\
\hline
\end{tabular}

Table 2. Treatment Results

\begin{tabular}{|c|c|c|c|}
\hline \multicolumn{2}{|c|}{$\begin{array}{c}\text { 3-Day } \\
\text { (n=24) }\end{array}$} & $\begin{array}{c}\text { 7-Day } \\
\text { (n=20) }\end{array}$ & P-Value \\
\hline \hline $\begin{array}{c}\text { Persistent bacteriuria } \\
\text { (positive test of cure) }\end{array}$ & $6(25 \%)$ & $2(10 \%)$ & 0.19 \\
\hline Recurrent UTI & $2(8 \%)$ & $1(5 \%)$ & 0.57 \\
\hline Pyelonephritis & $5(21 \%)$ & $6(30 \%)$ & 0.48 \\
\hline $\begin{array}{c}\text { Days in hospital for those } \\
\text { subjects diagnosed with } \\
\text { pyelonephritis (range) }\end{array}$ & $2.80 \pm 0.84(2-4)$ & $2.83 \pm 1.16(2-5)$ & 0.40 \\
\hline
\end{tabular}

Obstetrical and neonatal outcome measures of both groups did not differ significantly (Table 3). Both groups delivered at a similar gestational age, with a similar birth weight. One preterm birth occurred in the 7-day treatment group; an intrauterine fetal demise occurred at 23.6 weeks due to multiple congenital anomalies. It is unlikely that this occurrence was related to infectious causes or to the duration of treatment. In addition, the subject had successfully cleared the initial bacterium with treatment and did not experience any further infection during her pregnancy. Admissions to the Neonatal Intensive Care Unit (NICU) also did not differ between the two groups. The vast majority of these admissions were for suspected sepsis.

Table 3. Neonatal Outcomes

\begin{tabular}{|l|c|c|c|}
\hline \multicolumn{1}{|c}{} & \multicolumn{1}{c}{$\begin{array}{c}\text { 3-Day } \\
(\mathbf{n}=\mathbf{2 4})\end{array}$} & $\begin{array}{c}\text { 7-Day } \\
(\mathbf{n}=\mathbf{2 0})\end{array}$ & P-Value \\
\hline \hline $\begin{array}{l}\text { Gestational age at delivery } \\
\text { (weeks) }\end{array}$ & $38.6 \pm 1.6$ & $37.0 \pm 4.1$ & 0.08 \\
\hline $\begin{array}{l}\text { Delivery < 34 weeks } \\
\text { (range) }\end{array}$ & $0(36.4-40.6)$ & $1(29.2-42.0)$ & 0.47 \\
\hline $\begin{array}{l}\text { Birth weight } \\
\text { (grams) }\end{array}$ & $3262 \pm 489$ & $2909 \pm 969$ & 0.13 \\
\hline NICU admission & 5 & 4 & 0.32 \\
\hline
\end{tabular}

\section{DISCUSSION}

The key finding of our limited data indicates that 3-day treatment of ASB in pregnancy is statistically equivalent to 7-day treatment; however, the clinical implications of a $25 \%$ chance of persistence compared to a $10 \%$ chance are marked and would suggest that this regimen is inferior to a longer duration of treatment. No significant difference was noted in the occurrence of persistent bacteriuria, recurrence, or in progression to pyelonephritis. Neonatal morbidities were also similar, including gestational age at delivery, birth weight and NICU admissions.

Our results should further be considered in light of the recent published World Health Organization trial evaluation 1-day vs 7-day nitrofurantoin treatment for asymptomatic bacteriuria in pregnancy. In that investigation, 778 women were recruited for participation in this international, multicenter trial. Three hundred eighty-six women were randomly assigned to 1-day treatment, and 392 women were assigned to 7-day treatment. Bacteriologic cure rates at treatment day 14 were $75.7 \%$ and $86.2 \%$ for 1 -day and 7 -day regimens, respectively. The cure rate difference was $-10.5 \%$ (95\% CI $16.1 \%$ to $-4.9 \%$ ). Interestingly, there were also differences in neonatal outcomes such that mean birth weight and mean gestational age at delivery were significantly lower in the 1day regimen group. The authors concluded that pregnant women with ASB should be treated with 7-days of nitrofurantoin [18].

Earlier studies examining short course therapy for ASB in pregnancy focused on single dose regimens [25], which have been associated with higher rates of early recurrence by the original uropathogen $[26,27]$. This is postulated to be secondary to incomplete eradication of the bacteria from the urinary tract, and potentially high colony counts from enteric repositories. Other "short course" treatment regimes include four- and five-day antimicrobial courses which were hypothesized to be more effective at complete eradication of bacteria from the urinary tract than the single dose regimen [26]. While randomized trials conclude that these regimens are effective, the studies, like this one, are limited by small sample sizes.

We acknowledge the limitations of this investigation. Although the results support prior studies indicating the usefulness of short-course antimicrobial therapy in ASB in pregnancy, by its nature as a pilot investigation, the current study was underpowered to exclude the role of chance influencing the results; comparability or lack thereof of the treatment groups was not fully proven [28]. Based on the results of our pilot investigation, using an alpha-error of 0.05 , for $90 \%$ power, a total of 146 women in either treatment arm would be needed.

Our choice of antibiotics was also based on institutional history; a different antibiotic may have demonstrated greater efficacy [19]. Our diagnostic criterion for ASB was based on a single culture. We acknowledge that the recommendation of the IDSA [29] for the diagnosis of ASB is two urine cultures with colony counts which exceed $100,000 / \mathrm{mL}$. Because of logistical difficulties that would require subjects to return to the clinic for repeat cultures, we based our diagnosis on a single sample. This may have led to over diagnosis of ASB and also led to the atypical assortment of uropathogens identified. Because the repeat urine cultures were not obtained in some subjects for up to four weeks after diagnosis of ASB, it is possible that some of these cases represented colonization, rather than persistence or lack of 
clinical effect of the prescribed antibiotic. Most TOCs, however, were obtained within two weeks of diagnosis. Some of the organisms may have been skin contaminants because of our urine collection methods. But, given randomization, we would expect equal distribution between the groups. Other collection methods such as urinary catheterization would not have been practical. Lastly, more than $25 \%$ of the subjects dropped out of the investigation, with proportionately more from the 3-day group than the 7day group. Ideally, this would have not occurred. Nonetheless, we believe our study has merit as the only description of its kind for the treatment of ASB in pregnancy, and provides the basis for larger trials in the future.

Proven short-course therapy might prove beneficial in decreasing overall healthcare costs as well as improving patient compliance [30]. Currently, the annual direct cost of urinary tract infections in the United States approximates \$1 billion, emphasizing the healthcare burden incurred by the 8 million women annually seeking healthcare for evaluation of urinary tract infections [31-33]. In light of the compelling results from the WHO trial of 1-day vs 7-day nitrofurantoin treatment for ASB which favor the longer course, future investigations may focus on differing choices of antibiotics rather than on the duration of antimicrobial treatment for ASB in pregnancy.

\section{CONCLUSION}

Based on this limited investigation, 3-day antimicrobial therapy appears inferior to 7-day treatment for ASB in pregnancy.

\section{ACKNOWLEDGEMENTS}

\begin{tabular}{|l|c|c|c|}
\hline Contribution & P. Mittal & V. Pan & D.A. Wing \\
\hline Concept & & $\mathrm{X}$ & $\mathrm{X}$ \\
\hline Design & $\mathrm{X}$ & $\mathrm{X}$ & $\mathrm{X}$ \\
\hline Data Acquisition & $\mathrm{X}$ & $\mathrm{X}$ & $\mathrm{X}$ \\
\hline $\begin{array}{l}\text { Data Analysis and } \\
\text { Interpretation }\end{array}$ & $\mathrm{X}$ & $\mathrm{X}$ & $\mathrm{X}$ \\
\hline Manuscript Draft & $\mathrm{X}$ & $\mathrm{X}$ & $\mathrm{X}$ \\
\hline Manuscript Review & & & \\
\hline
\end{tabular}

\section{REFERENCES}

[1] Whalley PJ. Significance of asymptomatic bacteriuria detected during pregnancy. JAMA 1965; 193: 879-81.

[2] Krcmery S, Hromec J, Demesova D. Treatment of lower urinary tract infection in pregnancy. Int $\mathbf{J}$ Antimicrob Agents 2001; 17: 279-82.

[3] Millar LK, Cox SM. Urinary tract infections complicating pregnancy. Infect Dis Clin North Am 1997; 11: 13-26.

[4] Connolly A, Thorp JM. Urinary tract infections in pregnancy. Urol Clin North Am 1999; 26: 779-87.
[5] Christensen F. Which antibiotics are appropriate for treating bacteriuria in pregnancy? J Antimicrob Chemother 2000; 46(Suppl 1): 29-34.

[6] Le J, Briggs FF, McKeown A, Bustillo G. Urinary tract infections during pregnancy. Ann Pharmacother 2004; 38: 1692-701.

[7] Lenke RR, VanDorsten JP, Schifrin BS. Pyelonephritis in pregnancy: A prospective randomized trial to prevent recurrent disease evaluating suppressive therapy with nitrofurantoin and close surveillance. Am J Obstet Gynecol 1983; 146: 953-7.

[8] Romero R, Oyarzun E, Mazor M, Sirtori M, Hobbins JC, Bracken M. Meta-analysis of the relationship between asymptomatic bacteriuria and preterm delivery/low birth weight. Obstet Gynecol 1989; 73: 576-82.

[9] Mazor-Draz E, Levy A, Schlaeffer F, Sheiner E. Maternal urinary tract infection: Is it independently associated with adverse pregnancy outcome? J Matern Fetal Neonatal Med 2009; 22: 124 28.

[10] Villar J, Gulmezoglu AM, de Onis M. Nutritional and antimicrobial interventions to prevent preterm birth: An overview of randomized controlled trials. Obstet Gynecol Survey 1998; 53: 575-85.

[11] Kass EH. Bacteriuria and pyelonephritis of pregnancy. Arch Intern Med 1960; 105: 194-8.

[12] Rouse DJ, Andrews WW, Goldenberg RL, Owen J. Screening and treatment of asymptomatic bacteriuria of pregnancy to prevent pyelonephritis: A cost-effectiveness and cost-benefit analysis. Obstet Gynecol 1995; 86: 119-23.

[13] Smaill F. Antibiotics for asymptomatic bacteriuria in pregnancy. Cochrane Database Syst Rev 2001; 2: CD000490.

[14] Wing DA. Pyelonephritis. Clin Obstet Gynecol 1998; 41: 515-26.

[15] Mabie WC, Barton JR, Sibai B. Septic shock in pregnancy. Obstet Gynecol 1997; 90(4 Pt 1): 553-61.

[16] Graves CR. Acute pulmonary complications during pregnancy. Clin Obstet Gynecol 2002; 45: 369-76.

[17] Gilstrap LC, Ramin SM. Urinary tract infections during pregnancy. Obstet Gynecol Clin North Am 2001; 28: 581-91.

[18] Lumbiganon P, Villar J, Laopaiboon M, et al. World Health Organization Asymptomatic Bacteriuria Trial Group. One-day compared with 7-day nitrofurantoin for asymptomatic bacteriuria in pregnancy: A randomized controlled trial. Obstet Gynecol 2009; 113: 339-45.

[19] Smaill F, Vasquez JC. Antibiotics for asymptomatic bacteriuria in pregnancy. Cochrane Database Syst Rev 2007; (2): CD000490.

[20] Ovalle A, Levancini M. Urinary tract infections in pregnancy. Curr Opin Urol 2001; 11: 55-9.

[21] Hooton TM. Fluoroquinolones and resistance in the treatment of uncomplicated urinary tract infection. Int $\mathrm{J}$ Antimicrob Agents 2003; 22(Suppl 2): 65-72.

[22] Nicolle LE. Short-term therapy for urinary tract infection: Success and failure. Int J Antimicrob Agents 2008; Suppl 1: S40-5.

[23] Warren JW, Abrutyn E, Hebel JR, Johnson JR, Schaeffer AJ Stamm WE. Guidelines for antimicrobial treatment of uncomplicated acute bacterial cystitis and acute pyelonephritis in women. Infectious Diseases Society of America (IDSA). Clin Infect Dis 1999; 29: 745-58.

[24] Tan JS, File TM Jr. Treatment of bacteriuria in pregnancy. Drugs 1992; 44: 972-80.

[25] Jakobi P, Neiger R, Merzbach D, Paldi E. Single-dose antimicrobial therapy in the treatment of asymptomatic bacteriuria in pregnancy. Am J Obstet Gynecol 1987; 156: 1148-52.

[26] Villar J, Lydon-Rochelle MT, Gulmezoglu AM, Roganti A. Duration of treatment for asymptomatic bacteriuria during pregnancy. Cochrane Database Syst Rev 2000; 2: CD000491.

[27] Rubin RH, Shapiro DS, Andriole VT, Davis RJ, Stamm WE. Evaluation of new anti-infective drugs for the treatment of urinary tract infection. Infectious Diseases Society of America and the Food and Drug Administration. Clin Infect Dis 1992; 15(Suppl 1): S216-27.

[28] Jones B, Jarvis P, Lewis JA, Ebbutt AF. Trials to assess equivalence: The importance of rigorous methods. BMJ 1996; 313 : 36-9.

[29] Nicolle LE, Bradley S, Colgan R, Rice JC, Schaeffer A, Hooton TM. Infectious Diseases Society of America guidelines for the diagnosis and treatment of asymptomatic bacteriuria in adults. Clin Infect Dis 2005; 40: 643-54. 
[30] Grob PR. Antibiotic prescribing practices and patient compliance in the community. Scand J Infect Dis 1992; Suppl 83: 7-14.

[31] Foxman B, Barlow R, D'Arcy H, Gillespie B, Sobel JD. Urinary tract infection: Self-reported incidence and associated costs. Ann Epidemiol 2000; 10: 509-15.
[32] Foxman B. Epidemiology of urinary tract infections: incidence, morbidity, and economic costs. Dis Mon 2003; 49: 53-70.

[33] Schappert S. National ambulatory medical care survey: 1994 summary. Adv Data 1996; 10: 1-18.

Received: September 18, 2009

Revised: October 7, 2009

Accepted: October 16, 2009

(C) Mittal et al.; Licensee Bentham Open.

This is an open access article licensed under the terms of the Creative Commons Attribution Non-Commercial License (http://creativecommons.org/licenses/ by-nc/3.0/) which permits unrestricted, non-commercial use, distribution and reproduction in any medium, provided the work is properly cited. 